\title{
In Response: From Nath
}

\section{TO THE EDITOR:}

In the above letter to the editor, Roger Chou implies that the results of our trial (1) could be biased and concludes that, "It is difficult to see how the Nath et al trial could be taken as reliable evidence that radiofrequency denervation is effective." He further implies that, "...because the sham control group (which had higher baseline scores) had greater potential to experience improvement from baseline." However, Chou misunderstood as it was the active treatment group that had higher baseline scores of pain, not the sham group as was stated by him.

However, whether patients with more pain are more easily treated than those with less pain has not been studied; indeed the converse may well be true. This criticism is therefore unsubstantiated. The individual patient data are presented which illustrate the baseline and outcome measures for 1.) Generalized pain, 2.) Back pain, 3.) Leg pain and 4.) Global (perception of) Improvement. 


\section{Generalized Pain}

Active RF lesion

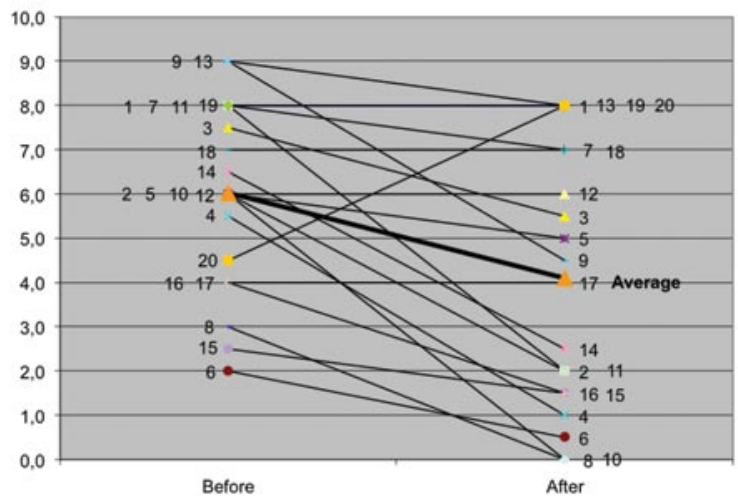

Sham RF lesion

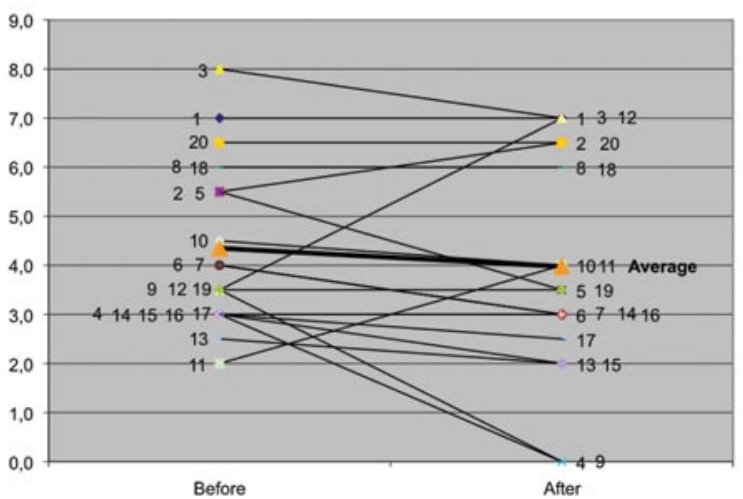

\section{Back Pain}

Active RF lesion

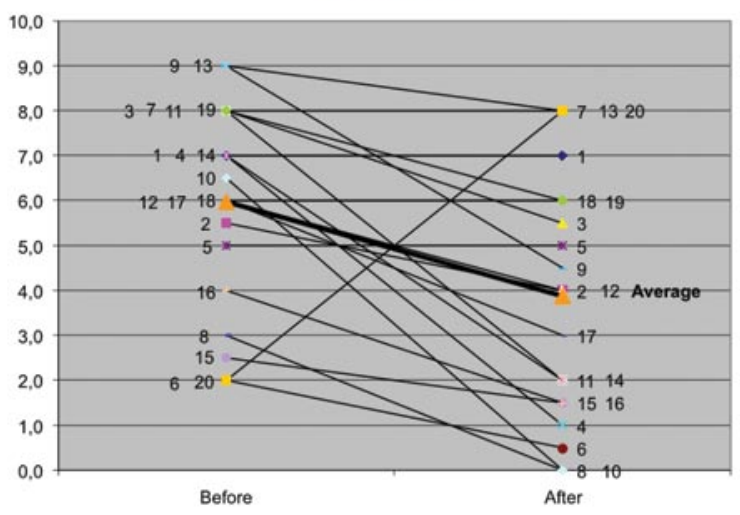

Sham RF lesion

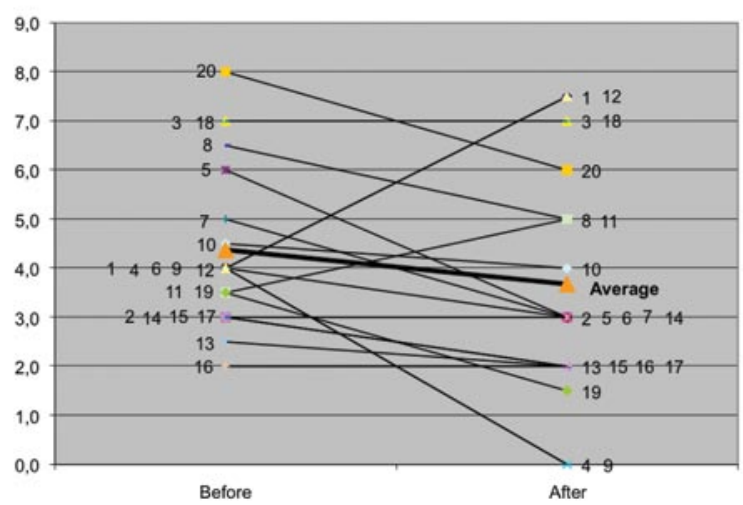

\section{Leg Pain}

Active RF lesion

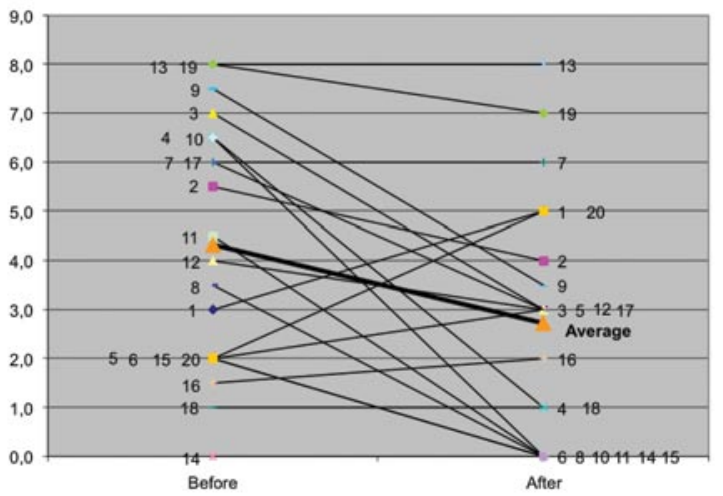

Sham RF lesion

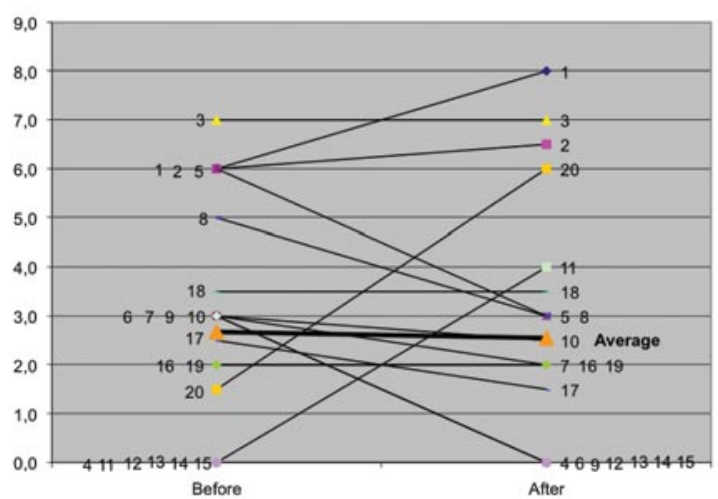




\section{Global Improvement}

Active RF lesion

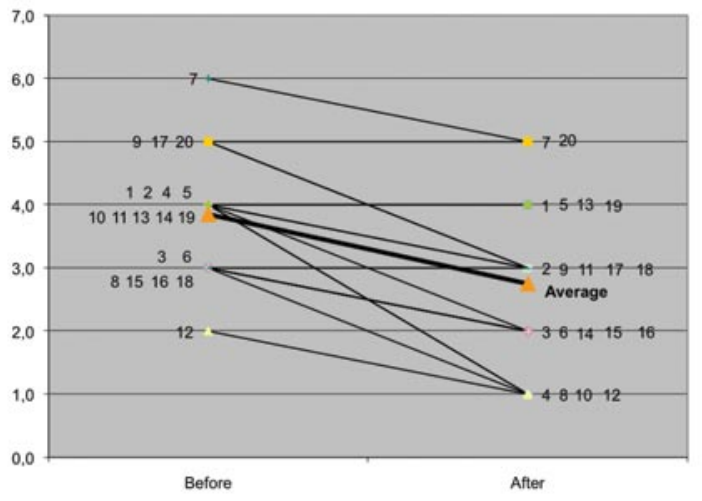

Sham RF lesion

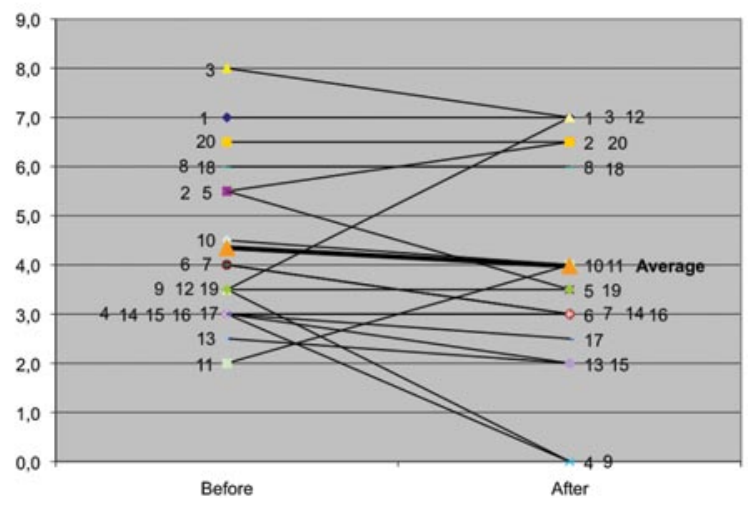

The graphs do not indicate any bias, as described by Chou. It was not as if patients, in the active group, with more severe pain responded to treatment better or more often than patients with less severe pain. $\mathrm{Pa}$ tients with all degrees of severity responded. Indeed, if anything, patients with more severe pain more often contributed to failures, than did patients with less severe pain.

Thus, Dr. Chou's criticism therefore is based on an abstract, theoretical principle that is not anchored to the actual data. Further, it amounts to a phantom designed to scare readers into discrediting lumbar RF. The phantom is not real.
Sherdil Nath, MD

Östra Esplanaden 6

90330 Umeå

Sweden

sherdil@hotmail.com

\section{References}

1. Nath S, Nath C, Pettersson K. Percutaneous lumbar zygapophysial (facet) joint neurotomy using radiofrequency current, in the management of chronic low back pain. Spine (Phila Pa 1976) 2008; 33:1291-1297. 